\title{
Long Non-Coding RNAs in Cancer: Diagnostic and Prognostic Value
}

Nina Hauptman and Damjan Glavač*

Department of Molecular Genetics, Institute of Pathology, Medical Faculty, University of Ljubljana, SI-1000 Ljubljana, Slovenia

\begin{abstract}
Long non-coding RNAs (IncRNAs) are transcripts of more than 200 nucleotides in length with limited protein coding potential were found to be pervasively transcribed and are associated with tumorigenesis due to their various functions in transcriptional, posttranscriptional and epigenetic mechanisms of gene regulation. IncRNAs are found to be differentially expressed in various cancers and associated with cancer development and progression, revealing their potential for diagnostic and prognostic biomarkers in cancer. In this review, we will summarize the most important IncRNAs, focusing on their diagnostic and prognostic potential in different cancers.
\end{abstract}

Keywords: Long non-coding RNAs; Cancer; Prognostic biomarkers; Gene expression

\section{Introduction}

Control of cell functions is regulated by gene expression, which depends on numerous proteins and non-protein coding RNAs (ncRNAs). ncRNAs have been identified as gene expression modulators, having different functions and targets [1,2]. Diverse classes of ncRNA are being studied. These molecules can be broadly classified into two groups, one being small non-coding RNAs, such as microRNAs, and the other being long non-coding RNAs(lncRNAs), subdivided into long intergenic lncRNAs, intronic lncRNAs, sense lncRNAs, antisense lncRNAs, bi-directional IncRNAs, promoter-associated lncRNAs, enhancer lncRNAs and natural antisense transcriptions (Figure 1).

Various cancer types exhibit deregulated levels of different lncRNAs. Some are deregulated in many types of cancer, while others are specific in expression in specific type of tissue. Therefore, lncRNA are an interesting field of new cancer biomarkers, for either a detection of a certain type of cancer, or for progression of cancer.

\section{IncRNA Valuable as Diagnostic and Prognostic Biomarkers}

HOX Antisense Intergenic RNA (HOTAIR) was shown to be deregulated in many types of cancers, including lung cancer, breast cancer, and cancers of the digestive tract [3]. HOTAIR is most valuable as prognostic biomarker, especially for identification of metastatic potential [4]. Upregulated expression was observed in patients with colorectal cancer, where a probable five-year overall survival is $55 \%$ compared to $80 \%$ in patients with lower levels of HOTAIR in tumor tissue [5]. Upregulation of HOTAIR was associated with lower survival rate and decrease of metastasis-free survival rate in patients with breast cancer, compared to those with lower expression values of HOTAIR [6]. In hepatocellular carcinoma (HCC) and HCC patients with liver transplantation, the expression of HOTAIR when compared with ordinary liver tissue are upregulated. Expression levels of HOTAIR can also be used as an independent prognostic marker for HCC recurrence and lower survival rate [7]. HOTAIR can be used as potential biomarker for the existence of lymph node metastasis in HCC [8]. These researches support the role of HOTAIR lncRNA as a metastazation biomarker.

MALAT1 was the first lncRNA associated with high metastatic potential and poor patient prognosis during a comparative screen of non-small cell lung cancer patients with or without metastatic tumors [9]. Although it is expressed in normal tissues but it was found to be deregulated in many cancers. Its overexpression was observed in colorectal cancer, lung cancer, gastric cancer, prostate cancer and HCC [9-14]. It has been shown that increased expression of MALAT1 can be used as a prognostic marker for HCC patients following liver transplantation [15]. Research on colorectal cancer showed significant inverse correlation with disease-free and overall survival. Patients with high expression levels were compared to those patients, which expressed lower levels of MALAT1 expression. For higher levels the five-year survival rate was $48 \%$ and overall survival was $67 \%$, for lower levels the rates were $67 \%$ and $85 \%$, respectively [16]. Expression levels of MALAT1 have a prognostic potential, correlating its levels to poor outcome for patients with cancer [17].

Prostate Cancer Associated 3 (PCA3) has been shown to be upregulated in prostate cancer tissue $[18,19]$. IT was also shown that PCA3 is highly specific for prostate cancer, since it was not detected in other types of cancer [18]. The evidence suggests that PCA3 is specific biomarker for prostate cancer.

PCGEM1 is also a prostate-specific transcript, and expressed just in prostate. The expression is upregulated in prostate cancer tissue, compared to normal [20]. Although specific for prostate cancer, there has not been found an association between levels of PCGEM1 and patient survival rates [21].

Urothelial cancer associated 1 (UCA1) was found to be a potential biomarker for bladder cancer [22]. In tumor tissue expression of UCA1 is upregulated, and it can be detected in cellular sediment with sensitivity of $80.9 \%$ for determining the disease diagnosis. It also allows discrimination of urothelial cancer from other urinary tract diseases, such as renal cell carcinoma, upper urinary tract restriction, and neurogenic bladder, and others, with overall specificity of $91.8 \%$ [22]. It has been shown that even higher expression was detected in urine in progressive stage of urothelial cancer [23].

Highly upregulated in liver cancer (HULC) lncRNA exhibits high levels of expression in liver cancer compared to normal hepatic tissue. In cancers with higher Edmondson grade classification the expression of HULC is even higher, and correlates with disease aggressiveness, which is supporting the potential of HULC as biomarker of prognosis [24].

*Corresponding author: Damjan Glavač, Department of Molecular Genetics, Institute of Pathology, Medical Faculty, University of Ljubljana, SI-1000 Ljubljana, Slovenia, Tel: +38615437180; Fax: +38615437181; E-mail: damjan.glavac@mf.uni-lj.si

Received June 15, 2016; Accepted June 21, 2016; Published June 24, 2016

Citation: Hauptman N, Glavač D (2016) Long Non-Coding RNAs in Cancer: Diagnostic and Prognostic Value. Med chem (Los Angeles) 6: 422-424. doi:10.4172/2161-0444.1000379

Copyright: (c) 2016 Hauptman N, et al. This is an open-access article distributed under the terms of the Creative Commons Attribution License, which permits unrestricted use, distribution, and reproduction in any medium, provided the original author and source are credited. 


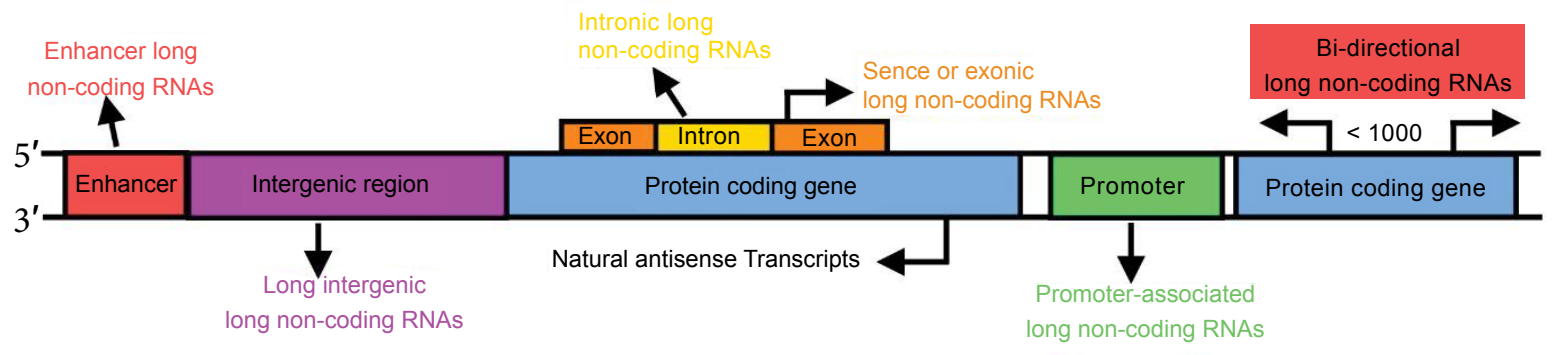

Figure 1: Illustrative representation of biogenesis of IncRNA. Various regions on the genome can produce different IncRNA, such as enhancer IncRNA, long intergenic IncRNA, intronic IncRNA, sense IncRNA, natural antisense transcripts, promoter-associated IncRNA and bi-directional IncRNA.

\begin{tabular}{|c|c|c|c|}
\hline IncRNA & Cancer Types & Regulation & Diagnostic and/or prognostic value \\
\hline HOTAIR & Breast, Lung, Colorectal, Hepatocellular & $\uparrow$ & Metastazation biomarker \\
\hline MALAT1 & Colorectal, Lung, Gastric, Prostate, Hepatocellular & $\uparrow$ & Poor outcome \\
\hline PCA3 & Prostate & $\uparrow$ & Specific for prostate cancer \\
\hline PCGEM1 & Prostate & $\uparrow$ & Specific for prostate cancer \\
\hline UCA1 & Urothelial & $\uparrow$ & Specific for urothelial cancer \\
\hline HULC & Hepatocellular & $\uparrow$ & Prognosis in hepatocellular cancer \\
\hline SRA & Breast, Uterus, Ovary & [33-35] \\
\hline MEG3 & Brain & $\uparrow$ & Steroid-based tumors \\
\hline
\end{tabular}

Table 1: Diagnostic and prognostic value of cancer associated IncRNAs (adapted from Ref. [29] and Ref. [30]).

SRA is a coactivator for steroid receptors acts as an ncRNA discovered within the nucleus and protoplasm. SRA regulates process mediated by means of steroid receptors through complexing with proteins additionally containing steroid receptor coactivator one (SRC1) [25]. The SRA1 component encrypts a peptide molecule that acts as a coactivator and corepressor [26]. SRA levels are discovered to be upregulated in breast tumors, where it can be assumed that increased SRA levels have effect on the steroid receptors', in turn contributing to breast tumorigenesis. Whereas the expression of SRA in average tissues is low, it is particularly up-regulated in tumors of the breast, uterus and ovary. This proofs that SRA might have capabilities of a biomarker of steroid-based tumors [25].

Maternally Expressed Gene three (MEG3) was found to be downregulated in glioma and, is highly expressed in normal brain tissue. It has been associated with prolonged survival of patients with glioblastoma multiforme (GBM). Overexpression of MEG3 increased apoptosis and inhibited glioma cell proliferation [27,28]. Following this evidence MEG3 has the potential for prognostic biomarker.

We have summarized all the important lncRNA with potential biomarker capabilities in Table 1.

\section{Conclusions}

The potential of lncRNA is enormous, from diagnostics to therapy. From potential of being isolated from bodily fluids, to higher sensitivity following technological improvements, lncRNA may be recognized as cancer biomarkers with true diagnostic and prognostic value [36]. The strong correlations found thus far between expression deregulation of lncRNA and cancer initiation and progression, have been the basis of prediction for targeted therapies. It was shown that lncRNAs could act as regulator of the expression of miRNA, proposing that by targeted therapy, expression of both miRNA and lncRNA can be modified [37]. While few miRNA therapeutics have been already submitted to clinical trials (miR-34, let-7), the lncRNA therapy strategies are still being developed. Inactivation of lncRNA is possible through inhibition of active site or through disruption of secondary structure, leading to loss of function. Further research on structure and functions of lncRNA will uncover more possibilities for diagnostic and therapeutic possibilities of lncRNA.

\section{References}

1. Mattick JS, Makunin IV (2006) Non-coding RNA. Hum Mol Genet 15 Spec No 1: R17-29.

2. Kaikkonen MU, Lam MT, Glass CK (2011) Non-coding RNAs as regulators of gene expression and epigenetics. Cardiovasc Res 90: 430-440.

3. Yu X, Li Z (2015) Long non-coding RNA HOTAIR: A novel oncogene (Review) Mol Med Rep 12: 5611-5618.

4. Cai B, Wu Z, Liao K, Zhang S (2014) Long noncoding RNA HOTAIR can serve as a common molecular marker for lymph node metastasis: a meta-analysis. Tumour Biol 35: 8445-8450.

5. Kogo R, Shimamura T, Mimori K, Kawahara K, Imoto S, et al. (2011) Long noncoding RNA HOTAIR regulates polycomb-dependent chromatin modification and is associated with poor prognosis in colorectal cancers. Cancer Res 71: 6320-6326.

6. Gupta RA, Shah N, Wang KC, Kim J, Horlings HM, et al. (2010) Long noncoding RNA HOTAIR reprograms chromatin state to promote cancer metastasis. Nature 464: 1071-1076.

7. Morey L, Helin K (2010) Polycomb group protein-mediated repression of transcription. Trends Biochem Sci 35: 323-332.

8. Geng YJ, Xie SL, Li Q, Ma J, Wang GY (2011) Large intervening non-coding RNA HOTAIR is associated with hepatocellular carcinoma progression. J Int Med Res 39: 2119-2128.

9. Ji P, Diederichs S, Wang W, Böing S, Metzger R, et al. (2003) MALAT-1, novel noncoding RNA, and thymosin beta4 predict metastasis and survival in early-stage non-small cell lung cancer. Oncogene 22: 8031-8041.

10. Hutchinson JN, Ensminger AW, Clemson CM, Lynch CR, Lawrence JB, et al. (2007) A screen for nuclear transcripts identifies two linked noncoding RNAs associated with SC35 splicing domains. BMC Genomics 8: 39.

11. Guffanti A, lacono M, Pelucchi $P$, Kim N, Soldà G, et al. (2009) A transcriptional sketch of a primary human breast cancer by 454 deep sequencing. BMC Genomics 10: 163.

12. Yamada K, Kano J, Tsunoda H, Yoshikawa H, Okubo C, et al. (2006) Phenotypic characterization of endometrial stromal sarcoma of the uterus. Cancer Sci 97: 106-112.

13. Lin R, Maeda S, Liu C, Karin M, Edgington TS (2007) A large noncoding RNA 
Citation: Hauptman N, Glavač D (2016) Long Non-Coding RNAs in Cancer: Diagnostic and Prognostic Value. Med chem (Los Angeles) 6: $422-424$. doi:10.4172/2161-0444.1000379

is a marker for murine hepatocellular carcinomas and a spectrum of human carcinomas. Oncogene 26: 851-858.

14. Luo JH, Ren B, Keryanov S, Tseng GC, Rao UN, et al. (2006) Transcriptomic and genomic analysis of human hepatocellular carcinomas and hepatoblastomas. Hepatology 44: 1012-1024.

15. Lai MC, Yang Z, Zhou L, Zhu QQ, Xie HY, et al. (2012) Long non-coding RNA MALAT-1 overexpression predicts tumor recurrence of hepatocellular carcinoma after liver transplantation. Med Oncol 29: 1810-1816.

16. Zheng HT, Shi DB, Wang YW, Li XX, Xu Y, et al. (2014) High expression of IncRNA MALAT1 suggests a biomarker of poor prognosis in colorectal cancer. Int J Clin Exp Pathol 7: 3174-3181.

17. Zhang J, Zhang B, Wang T, Wang H (2015) LncRNA MALAT1 overexpression is an unfavorable prognostic factor in human cancer: evidence from a metaanalysis. Int J Clin Exp Med 8: 5499-5505.

18. Bussemakers MJ, van Bokhoven A, Verhaegh GW, Smit FP, Karthaus HF, et al. (1999) DD3: a new prostate-specific gene, highly overexpressed in prostate cancer. Cancer Res 59: 5975-5979.

19. Hessels D, Schalken JA (2009) The use of PCA3 in the diagnosis of prostate cancer. Nat Rev Urol 6: 255-261.

20. Srikantan V, Zou Z, Petrovics G, Xu L, Augustus M, et al. (2000) PCGEM1, a prostate-specific gene, is overexpressed in prostate cancer. Proceedings of the National Academy of Sciences 97: 12216-12221.

21. Prensner JR, Sahu A, Iyer MK, Malik R, Chandler B, et al. (2014) The IncRNAs PCGEM1 and PRNCR1 are not implicated in castration resistant prostate cancer. Oncotarget 5: 1434-1438.

22. Wang XS, Zhang Z, Wang HC, Cai JL, Xu QW, et al. (2006) Rapid Identification of UCA1 as a Very Sensitive and Specific Unique Marker for Human Bladde Carcinoma. Clinical Cancer Research 12: 4851-4858.

23. Srivastava AK, Singh PK, Rath SK, Dalela D, Goel MM, et al. (2014) Appraisal of diagnostic ability of UCA1 as a biomarker of carcinoma of the urinary bladder. Tumour Biol 35: 11435-11442.

24. Xie H, Ma H, Zhou D (2013) Plasma HULC as a Promising Novel Biomarker for the Detection of Hepatocellular Carcinoma. BioMed Research International 2013: ID 136106

25. Lanz RB, Chua SS, Barron N, Söder BM, DeMayo F, et al. (2003) Steroid receptor RNA activator stimulates proliferation as well as apoptosis in vivo. Mol Cell Biol 23: 7163-7176.
26. Chooniedass-Kothari S, Vincett D, Yan Y, Cooper C, Hamedani MK, et al. (2010) The protein encoded by the functional steroid receptor RNA activator is a new modulator of ER alpha transcriptional activity. FEBS Lett 584: 11741180 .

27. Wang $P$, Ren $Z$, Sun $P$ (2012) Overexpression of the long non-coding RNA MEG3 impairs in vitro glioma cell proliferation. J Cell Biochem 113: 1868-1874.

28. Zhang X, Sun S, Pu JK, Tsang AC, Lee D, et al. (2012) Long non-coding RNA expression profiles predict clinical phenotypes in glioma. Neurobiol Dis 48: 1-8.

29. Hauptman N, GlavaÄ D (2013) Long non-coding RNA in cancer. Int J Mol Sci 14: $4655-4669$.

30. Silva A, Bullock M, Calin G (2015) The Clinical Relevance of Long Non-Coding RNAs in Cancer. Cancers 7: 2169-2182.

31. Svoboda M, Slyskova J, Schneiderova M, Makovicky P, Bielik L, et al. (2014) HOTAIR long non-coding RNA is a negative prognostic factor not only in primary tumors, but also in the blood of colorectal cancer patients. Carcinogenesis 35 1510-1515.

32. Weber DG, Johnen G, Casjens S, Bryk O, Pesch B, et al. (2013) Evaluation of long noncoding RNA MALAT1 as a candidate blood-based biomarker for the diagnosis of non-small cell lung cancer. BMC Res Notes 6: 518.

33. Xue WJ, Ying XL, Jiang JH, Xu YH (2014) Prostate cancer antigen 3 as a biomarker in the urine for prostate cancer diagnosis: a meta-analysis. J Cancer Res Ther 10 Suppl: C218-221.

34. Merola R, Tomao L, Antenucci A, Sperduti I, Sentinelli S, et al. (2015) PCA3 in prostate cancer and tumor aggressiveness detection on 407 high-risk patients: a National Cancer Institute experience. Journal of Experimental \& Clinical Cancer Research 34: 1-6.

35. Chevli KK, Duff M, Walter P, Yu C, Capuder B, et al. (2014) Urinary PCA3 as a predictor of prostate cancer in a cohort of 3,073 men undergoing initial prostate biopsy. J Urol 191: 1743-1748.

36. Mäbert K, Cojoc M, Peitzsch C, Kurth I, Souchelnytskyi S, et al. (2014) Cancer biomarker discovery: Current status and future perspectives. International Journal of Radiation Biology 90: 659-677.

37. Wang X, Li M, Wang Z, Han S, Tang X, et al. (2015) Silencing of long noncoding RNA MALAT1 by miR-101 and miR-217 inhibits proliferation, migration, and invasion of esophageal squamous cell carcinoma cells. J Biol Chem 290: 39253935 . 